\title{
Selective information processing
}

\author{
STEPHEN P. HEPLER \\ Department of Mathematics, Computer Science Section, Wayne State University, \\ Detroit, Michigan 48202
}

\begin{abstract}
Past studies have shown that in certain tasks, subjects are not able to selectively attend to specific areas of a visual display even if instructed to do so. Yet, a more recent study (Graves, 1976) has used the concept of selective attention to explain the difference between the number of items processed in forced choice detection tasks and the number processed in full report tasks. Graves proposed that only identification processing is necessary in the detection task, while both identification and position processing are required in the full report task. A problem with Graves' task is that it requires memory searching after stimulus presentation, probably reducing the predicted number of items processed. The experiment reported here utilized partially filled arrays and required responses based upon only positional processing, or only identification processing, or both types of processing. In direct contradiction to Graves' conclusions, the results showed that although subjects could inhibit identification processing while engaged in positional processing, the reverse was not true. In addition, positional processing was shown to be faster than identification processing.
\end{abstract}

Two distinct features of the human memory system have been distinguished: structural components and control processes (Atkinson \& Shiffrin, 1968). While the structural components refer to the permanent features of the system, the control processes are those processes the subject selects and actively uses to aid performance on a given task. It is known, for example, that subjects group similar items together for study, and that in some instances, subjects will combine some pieces of information into a larger unit and study the unit rather than the individual items. While these processes have been clearly identified, other processes have been shown not to be under the subject's control. Shiffrin has extensively studied the concept of attention and concluded that, in the perceptual system, subjects cannot selectively attend to certain areas of a display even if instructed to do so. If attention is a control process, subjects should be able to attend to each item independently of the other items during a sequential presentation of an informational display, while all items would compete for attention during a simultaneous presentation. Therefore, performance should be superior on the sequential presentation trials. However, the results in this type of experiment have shown that performance after simultaneous presentation is equal or superior to performance after sequential presentation (Shiffrin \& Gardner, 1972).

Another type of experiment concerning subjects' ability to attend to only a portion of a stimulus was reported by Shiffrin, McKay, and Shaffer (1976). They conducted an experiment in which subjects were shown a 7 by 7 array of dots in which at most one dot was missing. Subjects were then shown another 7 by 7 array of dots with one missing and had to determine if the missing dot was present or absent in the original stimulus. Subjects were told that all 49 positions, or the centermost 9 positions, or only the center position would be tested. Thus in the different conditions, subjects could selectively attend to only that portion of the stimulus that could be tested. Surprisingly, they found no difference in performance on tests of the centermost dot in the three experimental conditions and cited this as further evidence of the lack of an attention mechanism in perceptual memory. Graves (1976), however, has proposed a change in processing strategies, similar to the concept of attention, to explain the different estimates of items processed in a forced choice detection task and a full report task. In an experiment by Estes and Taylor (1964), subjects were simultaneously shown an array of letters and were asked to report which one of two previously specified letters was present in the stimulus. They concluded from the results that subjects had processed approximately nine items. In an experiment by Sperling (1960), subjects were shown an array of letters and asked to reproduce as much of the array as possible. In this experiment, subjects typically reported an average of 4.5 letters in the correct array position.

Due to the problems of comparing data from two distinctly different tasks, Graves (1976) proposed a new task combining the essential features of both of the previous tasks. While he also used a forced choice detection task, he did not specify the two critical letters until after the stimulus presentation. From the results, Graves also estimated that about nine items had been processed.

In a second experiment, Graves had his subjects report not only the critical letter that appeared in the stimulus, but also the location in the stimulus where 
it appeared. In this experiment the estimated number of letters in the correct stimulus location available to subjects was 4.45 , a number very comparable to the average number of items reported in the Sperling (1960) experiment.

From these experiments Graves concluded that different types of stimulus processing were required in each of the two tasks and that this accounted for the different estimates of items processed. In the detection task, only identification processing was required. As a result, a large number of items were processed. In the full report task, however, positional processing was also required, and this processing competed with identification processing, reducing the total number of items processed for identification.

Graves' (1976) results and conclusions raise two important questions. Since the critical letters are not given until after the stimulus presentation, the subject must wait until after the stimulus has been presented and search the stimulus representation stored in memory. While the search is being conducted, items are undoubtedly being lost from memory, thereby reducing the number of items available. It could be argued that a similar phenonemon occurs in the standard full report task. The very act of responding interferes with rehearsal of items, and items originally perceived may become unavailable for later report. It is in fact because of this problem that partial report tasks have been utilized (Sperling, 1960). In these tasks, subjects report only a portion of the stimulus but are unaware of the portion to be reported until after stimulus presentation. These reports have estimated that as many as nine items are available to subjects immediately after stimulus presentation, a number very comparable to the Estes and Taylor (1964) estimate. Thus it is still not clear that subjects are engaging in different strategies in the two tasks. It is possible that the stimulus is processed to the same degree in both experiments. It may be that the difference in results is attributable to the processes involved in the memory search prior to the response generation. In the first experiment, only the identification information need be considered during the memory search, while both types of information are considered in the second experiment. It is necessary to design a full report task in which subjects can employ either both position and identification processing or only identification processing during the stimulus presentation and compare the results. This would avoid the major problem with Graves' (1976) task: possible memory loss due to the searching required after the stimulus presentation has been completed.

The second question raised by Graves' (1976) conclusions is, if subjects can inhibit positional processing in favor of identification processing, is the reverse true? In the standard full report task, positional processing may be an automatic and a rather simple process since each array position is always full. Thus the need for positional processing may be minimized by studying row by row or column by column. It is necessary to design a full report task in which positional processing is not necessarily automatic and to then require a response that is based solely on positional processing.

The task used in the experiment reported here is the standard full report task with two important exceptions. First, the stimulus array was only partially filled, with empty positions randomly included in the array. Second, three types of responses were used. Subjects were asked to report only the positions in the array that were not empty, or only the letters that appeared in the array, or the letters in their correct position in the array. Hereafter, these will be referred to as location only, letter only, and letter and location, respectively. Location only requires only positional processing, while letter only requires only identification processing. The last condition requires both types of processing.

The purpose of the present experiment was not only to answer the questions raised by Graves' (1976) conclusions, but also to extend the conclusions of Shiffrin (1975; Shiffrin \& Gardner, 1972; Shiffrin, Gardner, \& Allmeyer, 1973; Shiffrin \& Grantham, 1974; Shiffrin, McKay, \& Shaffer, 1976). While Shiffrin has shown that subjects are unable to selectively attend to one portion of a stimulus, it will now be possible to determine if subjects can selectively attend to different aspects of the same stimulus.

\section{METHOD}

\section{Subjects}

The subjects were 12 unpaid volunteer graduate and undergraduate students.

\section{Apparatus and Stimuli}

A Cambridge two-channel tachistoscope was used to present a stimulus field of 200-msec duration preceded and followed by the same noninformational field. The subject initiated the exposure sequence by pressing a button. View was binocular. The stimuli, arrays with three rows and four columns with six randomly selected cells containing characters and the remaining six cells empty, subtended a visual angle of 15 by 9 deg at the $30-\mathrm{cm}$ viewing distance. Vertical strokes and hyphens were used to outline the array. Characters were black uppercase type on a white ground illuminated by reflected light. The noninformational field was a $7.6 \times 5.7 \mathrm{~cm}$ rectangle with lines in a cross-hatch pattern. No fixation points were included in this field. Characters used were the 12 consonant letters $B$ through $P$.

\section{Procedure}

Prior to the actual data collection, each subject participated in 10 trials in each of the three experimental conditions. Each subject was then tested in all conditions with one condition tested each day. Six sequences of the three conditions were derived and two subjects were randomly assigned to each sequence. Each subject was informed of the required type of report at the beginning of each test day. Each test day consisted 
Table 1

Answer Types

\begin{tabular}{|c|c|c|}
\hline \multirow[b]{2}{*}{ Type } & \multicolumn{2}{|c|}{ Response } \\
\hline & Position Scoring & Letter Scoring \\
\hline & If the Position Was Full & If the Letter Was in Stimulus \\
\hline 1 & Filled in correctly & Reported in correct position \\
\hline 2 & Filled in incorrectly with letter in stimulus & Reported in incorrect but filled position \\
\hline 3 & Filled in incorrectly with letter not in stimulus & Reported in incorrect and empty position \\
\hline \multirow[t]{2}{*}{4} & Left empty & Not reported in answer \\
\hline & If the Position Was Empty & If the Letter Was Not in Stimulus \\
\hline 5 & Left empty & Not reported in answer \\
\hline 6 & Filled in & Reported in answer \\
\hline
\end{tabular}

of 30 test trials with the first 10 being ignored in the data analysis as warm-up trials. Answer sheets with the outline of a 3 by 4 array were used for subjects' responses. In the letteronly condition and in the letter-and-location condition, the 12 consonants were also included on the answer sheet, allowing subjects to either circle letters appearing in the stimulus or place them in the array. Two methods were used to score subjects' reports-one for location reporting and one for letter reporting. Letter-and-location condition reports were scored using both systems. The various possible answers are given in Table 1. Answers of Types 4 and 6 were the only incorrect answers in both scoring systems.

\section{RESULTS}

Since it could not be determined if an empty cell on an answer sheet represented a response or an ignored location, answers of Type 5 were not included in the data analysis. The average numbers of items correctly reported were 4.89 positions, 2.64 letters, 4.11 positions, and 2.52 letters for the three conditions, with the last two values representing the letter-andlocation condition performance using each scoring system. A two-way analysis of variance using a $p<.01$ rejection region showed a significant effect of conditions on the number of items reported $[F(3,912)=481.55$, $\mathrm{MSe}=.66]$, as well as a significant effect of subjects $[F(11,912)=55.78]$ and the interaction of the variables $[F(33,912)=5.74]$. To determine the strengths of these effects, $\omega^{2}$ (Hays, 1965) was calculated. It estimated that $45 \%, 19 \%$, and $6 \%$ of the variance in the number of items reported was accounted for by conditions, subjects, and the interaction, respectively.

Accurding to the scoring system given in Table 1, only answers of Types 4 and 6 are incorrect. However, by using these same systems to score letter-andlocation reports, some incorrect answers are scored as correct. Answers of Types 2 and 3 in both systems are incorrect in this condition. With this modification, letter-and-location condition reports have the same score under both systems and the average number of items reported in their correct array position in this condition drops to 2.10 . This value, along with the corresponding values for the location-only and letteronly conditions ( 4.89 positions and 2.64 letters), are significantly affected by the experimental conditions $\left[\mathrm{F}(2,684)=700.44, \omega^{2}=.54, \mathrm{MSe}=.75\right]$, as well as by subjects $\left[F(11,684)=32.97, \quad \omega^{2}=.14\right]$ and the interaction $\left[F(22,684)=6.05, \omega^{2}=.04\right]$.

Comparisons of the number of items reported between pairs of conditions were also significant: location only and letter only $\left[F(1,456)=882.82, \omega^{2}=.51\right.$, MSe $=.69]$, location only and letter and location $\left[\mathrm{F}(1,456)=1,185.50, \omega^{2}=.60, \mathrm{MSe}=.79\right]$, and letter only and letter and location $[F(1,456)=44.14$, $\left.\omega^{2}=.06, \mathrm{MSe}=.77\right]$.

At the conclusion of the experiment, each subject participated in a normal full report experiment. The stimuli were completely full 3 by 4 arrays of consonants. Subjects were only asked to reproduce as much of the stimulus as possible. The experiment consisted of 40 trials with the first 10 trials being ignored in the data analysis. All other aspects of the experiment were identical to the main experiment. Subjects reported an average of 2.52 letters in the correct array location.

\section{DISCUSSION}

The results clearly indicate that subjects do vary their study strategy depending upon the required type of report, and the effect of the type of strategy on the number of items reported is very strong, with more positions than letters being reported. Subjects claimed that they were able to process positional information while totally inhibiting identification processing, and this is supported by the data. While 4.89 of the 6 filled positions were correctly reported in the location-only condition, the average number of positions that were also reported with the correct letter included was .31 . Subjects also claimed the reverse was not true. They were not able to inhibit position processing and concentrate solely on identifying the letters in the letteronly condition. The data also support this observation. While 2.64 letters were correctly reported in the letteronly condition, the average number of letters reported in their correct array location in this condition was 1.66 , even though array location was not required in the report. Therefore, a total of at least 4.30 "items" 
(2.64 letters and 1.66 positions) were processed, compared to 5.20 items in the location-only condition and 4.20 items (2.10 letters and 2.10 positions) in the letter-and-location condition, indicating that position processing is faster than identification processing.

The data from the letter-and-location condition including answers of Types 2 and 3 indicated an average of 4.11 positions and 2.52 letters correctly reported. However, an average of only 2.10 letters were reported in the correct array location. One explanation for this is that positional information, identification information, and information linking together a letter and position could all be stored in memory. The latter information would then limit the number of items reported in the correct location. Again, it appears that position processing is much faster than identification processing, with identification processing being slightly faster than the process linking the two types of information together. This is what would be expected in a fixed-rate processing system, since a position being full or not full yields only 1 bit of information, while a specific letter appearing in the array yields 3.58 bits of information and the link between a full position and a specified letter yields 4.58 bits of information. This would also explain the data from the letter-only condition. The 1.66 letters reported in the correct position could be limited either by the number of positions processed, as previously hypothesized, or by the number of full positions and letters linked together. The latter explanation is more plausible, since position processing is most likely directly related to searching for a nonempty cell and this process always precedes identification processing.

All of the results presented support the concept of a fixed-rate information processing system in which the subject has some but not total control over the type of processing utilized in the different experimental tasks. The values from the full report experiment are quite comparable to the previously presented results. The difference in number of letters reported in the correct array location between the letter-and-location condition (2.10) and the full report experiment (2.52) can probably be attributed to search time. In the letterand-location condition, searching was necessary to find the next nonblank cell before the identification process could begin. This was not necessary in the full report experiment since every array position was full.

From this experiment, a model of information processing in this task can be proposed. (1) A detection process locates a nonempty array position. (2) Most of the positional information is processed automatically. For the location-only condition, no other processing is necessary and the detection process begins again. (3) For the letter-only and letter-and-location conditions, as well as for the full report experiment, the identification process begins. This is clearly under the subject's control but it is not known if the processing is done simultaneously with or immediately following the positional processing. Simultaneous processing could explain the average of .31 letters reported in the correct array location in the location-only condition. Since positional processing is much faster, identification processing would seldom be completed by the time positional processing is, and the detection process would begin again at that point. (4) A third process linking together positional information and identification information probably begins after the identification process is complete. This process again appears to be automatic since in the letter-only condition, letters were usually reported in their correct array position, even though this was not required by the response instructions. This process is limited, however, by the extent of identification processing that takes place.

Therefore, the proposed processing system includes four distinct processes, only two of which, the detection process and the identification process, are under direct subject control.

\section{REFERENCES}

Atkinson, R. C., \& Shiffrin, R. M. Human memory: A proposed system and its control processes. In $K$. $W$. Spence \& J. T. Spence (Eds.), The psychology of learning and motivation (Vol. 2). New York: Academic Press, 1968.

Estes, W. K., \& TAylor, H. A. A detection method and probabilistic models for assessing information processing from brief visual displays. Proceedings of the National Academy of Sciences, 1964, 52, 446-454.

Graves, R. E. Are more items identified than can be reported? Journal of Experimental Psychology: Human Learning and Memory, 1976, 2, 208-214.

Hays, W. Statistics. New York: Holt. Rinehart. \& Winston. 1965.

ShIFFRIN, R. M. The locus and role of attention in memory systems. In P. M. A. Rabbitt \& A. Dornic (Eds.), Attention and performance $V$. London: Academic Press, 1975.

ShIFFRIN, R. M., \& Gardner, G. T. Visual processing capacity and attentional control. Journal of Experimental Psychology, 1972, 93, 72-82.

Shiffrin, R. M., Gardner, G. T., \& Allmeyer, D. H. On the degree of attention and capacity limitations in visual processing. Perception \& Psychophysics, 1973, 14, 231-236.

ShiffRIN, R. M., \& GRANTham, D. W. Can attention be allocated to sensory modalities? Perception \& Psychophysics, 1974, 15, 460-474.

Shiffrin, R. M., McKay, D. P.. \& Shaffer, W. O. Attending to forty-nine spatial positions at once. Journal of Experimental Psychology: Human Perception and Performance, 1976, 2, 14-22.

Sperling, G. The information available in brief visual presentations. Psychological Monographs, 1960, 74(11, Whole No. 498).

(Received for publication January 25, 1977; revision accepted April 18, 1977.) 